\title{
Diagnosing collaboration in practice-based learning: Equality and Intra-individual variability of physical interactivity
}

\author{
Mutlu Cukurova ${ }^{1}$, Rose Luckin ${ }^{1}$, Eva Millán ${ }^{2}$, Manolis Mavrikis ${ }^{1}$, Daniel \\ Spikol $^{3}$
}

1. UCL Knowledge Lab, University College London, United Kingdom.

2. University of Málaga, Spain.

3. University of Malmö, Sweden.

\begin{abstract}
Collaborative problem solving (CPS), as a teaching and learning approach, is considered to have the potential to improve some of the most important skills to prepare students for their future. CPS often differs in its nature, practice, and learning outcomes from other kinds of peer learning approaches, including peer tutoring and cooperation; and it is important to establish what identifies collaboration in problemsolving situations. The identification of indicators of collaboration is a challenging task. However, students physical interactivity can hold clues of such indicators. In this paper, we investigate two non-verbal indexes of student physical interactivity to interpret collaboration in practice-based learning environments: equality and intra-individual variability. Our data was generated from twelve groups of three Engineering students working on open-ended tasks using a learning analytics system. The results show that high collaboration groups have member students who present high and equal amounts of physical interactivity and low and equal amounts of intra-individual variability.
\end{abstract}

Keywords: collaborative learning, problem-solving, indexes of physical interaction, behaviour patterns

\section{Introduction and Research Questions}

Collaborative problem solving (CPS), as a teaching and learning approach, has potential to provide opportunities to learners to practice and improve skills that are key to their future success in life. In addition to skill development, CPS can allow students to apply their knowledge, to explain it clearly to others, to synthesize it with fresh knowledge and knowledge from other subject areas[1]. CPS, therefore, helps learners acquire important subject knowledge. However, the research evidence from meta-level reviews shows that such improvement in skills and knowledge specific to CPS is rare. There is good evidence that well designed and managed peer learning, conducted by learners who have sufficient knowledge and skill and supported by teachers who also have the requisite skills, 
has a positive impact on learning including attainment in science [2], [3], mathematics [4], literacy [5] and upon learner attitudes [6]. However, this evidence is generated from meta-analyses and reviews of research based on studies examining peer-tutoring, cooperative learning, collaborative learning and collaborative problem-solving all together.

Although, these concepts are related, they are not synonymous. Clear distinctions were drawn between such group work pedagogies in theory (See for instance [7], [8]). These distinctions are seldom found in research papers and reviews of empirical work. However, they are important as different pedagogical approaches to students learning together, have different quality and degree of interaction and differ in their likelihood of achieving certain learning outcomes. For instance, collaborative and cooperative peer instructions are found to be more suited to students conceptual understanding, whereas peer-tutoring activities were found to be more appropriate for practice using concepts already acquired [9]. If the learning outcome of a session is to master a particular task that can be divided into sub-tasks, which can be achieved by individual students, cooperative learning, in which the learning group tackles the problems by dividing up the responsibility, may be more appropriate. Examples of such cooperative approaches involve jigsaw method [10] or the Sharan method [11].

On the other hand, if the task or the problem at hand cannot be achieved by any individual students on their own, then a collaborative learning approach is required, because it is particularly effective when solving problems that impose a high cognitive load [12]. As argued by Kirschner et al.[12] cognitive load theory suggests that collaborative learning may be effective for solving such problems and tasks that cannot be solved by any of the individuals in the group on their own, because it reduces load at the level of working memory within the minds of the individuals concerned. It was also showed in an experimental study [13] that collaborative learning was superior for high-complexity tasks, but inferior for low-complexity tasks. However, in practice, there is wide variation in group work with a lack of clarity regarding the impact of such variations on the intended learning outcomes and as argued by Damon and Phelps [7] "such lack of clarity places the credibility and educational usefulness of the entire enterprise of peers learning together at risk". This lack of clarity becomes even more ambiguous in practice-based teaching and learning environments due to the dynamic nature of the educational setting.

In this research study, we investigate two research questions aiming to provide more clarity to this variation in implementation of group learning in practicebased learning environments.

- RQ1. Can equality of physical interactivity and intra-individual variability be used as non-verbal indexes of collaboration in practice-based learning? Related to the first research question, our second research question is;

- RQ2. What amounts of physical interactivity and intra-individual variability represent collaboration in practice-based learning?

The proxies of student collaboration are often studied with verbal indexes $[14,15]$. Nevertheless, the work discussed in this paper is based on a European 
project (PELARS) in which one of the aims is to develop learning analytics tools for hands-on, open-ended STEAM (Science, Technology, Engineering and Maths teaching through the means of Art) learning activities using physical computing. The project has developed a software system that includes customized furniture with an integrated Learning Analytics System (LAS), that includes devices for tracking hands, faces and objects, and an Arduino platform with a visual webbased Integrated Development Environment (IDE), that captures information about interactions with these physical computing objects [16]. In this paper, we study non-verbal indexes of students physical interactivity. This is due to the challenges related to the collection and analysis of students' verbal indexes in dynamic practice-based learning environments.

\section{The importance of Collaboration and Problem-solving Skills}

One of the fundamental purposes of education is to support the teaching and learning process, so that each person is supported to achieve their potential. Although there are differences of opinion about what should be taught and how the teaching process should be conducted, there is some sense of agreement that the aim of education is to ensure that learners are equipped to meet their future needs, both in employment and generally in life. This perspective on education highlights the important role played by the skills that will be needed during the lifetime of students. More recently, these skills have been referred to as the 21st century skills [17], referring to the century in which current students will be living. There is no unanimously recognized definition of 21st century skills and various different suggestions exist. For example, the World Economic Forum (WEF) has proposed 16 skills [18], including collaboration and problem solving. A recent report from the UK Institute of Directors (2016) [19] stressed the need for schools to move away from the skills that are easiest to teach and test, because these are also the easiest to automate and therefore likely to be the least in demand in the workplace. They identified various skills as important including communication, collaboration and teamwork. These skills are essential for current and future work environments and they are key requirements of future education and training across the globe. Peer learning is intertwined with all the aforementioned key skills and is an increasingly common teaching approach to improve students 21st century skills. However, as we discussed in the introduction different practices of peer learning may lead to different learning outcomes, including different levels and types of skill development, as they involve different types and levels of student interactivity.

For instance, equality and mutuality are two indexes of student interactivity used to distinguish between three approaches to peer learning: peer tutoring, cooperative learning and collaborative learning. Equality refers to a situation where participants are equal in status and participate in a two-way dialogue taking direction from one another, while mutuality refers to a situation where high mutuality means that discourse is extensive, intimate and connected. As argued 
by Damon and Phelps [7] peer tutoring tends to foster dialogues that are relatively low on equality and varied in mutuality; cooperative learning foster ones that are relatively high in equality and low to moderate in mutuality; and peer collaboration fosters ones that are high in both. More recent researchers echo similar ideas. For instance, Dillenbourg et al. [20] use the concept of symmetry and argues that collaborative learning requires some sense of symmetry in terms of students knowledge and skills as well as their contribution to interactions. Identification of different approaches is key to create and apply learning tasks that achieve their intended learning outcomes (both in terms of skill development and knowledge) with more precision. One potential solution to identifying and differentiating these different approaches to students working together as a group in practice-based learning environments is to use indexes of students physical interactions. In this paper, we investigate two indexes of physical interactivity in order to identify unique features of collaborative problem solving in practice-based learning: a) equality of students physical interactivity, and b) intra-individual variability in students physical interactivity in practice-based learning activities.

The concept of equality is fairly self-explanatory and not novel to this research domain. However, students' intra-individual variability to our knowledge has not been investigated in the contexts of students' CPS in practice-based learning. As emphasised by various other researchers CPS tends to be inherently interactive, interdependent, and dynamic [21,22]. CPS can only occur if the students attempt to create a common ground about the problem/task they are dealing with [23]. The establishment of such shared understanding occurs through students communication and interaction with each other about the meaning of the problem/task. Creation of a common ground among group members is based on students ability to understand behaviours, cognitions, and attitudes of other participants and oneself and to translate this understanding into appropriate behaviour in social situations [24]. In this dynamic context, the establishment of a common ground involves continuous correction of students performance based on reactions of others during social exchanges [25]. This continuous correction and change in behaviours require a dynamic systems approach [26] to students physical interactions, as therefore we argue that the investigation of students' intra-individual variability may generate insights into students' CPS in practicebased learning activities.

\section{Methodology}

\subsection{Participants}

The study discussed in this paper involves 12 sessions of groups of 3 Engineering students at a European University (average age 20 years old, with 17 men and 1 woman). Students were volunteers to take part in the research and they had no formal CPS training in the past. However, they all declared that they have experience of working in groups as part of their university courses. Each student 
group used the PELARS project system hardware, software and desk over 3 days, to complete 3 open-ended tasks.

\subsection{The Task}

The students were introduced to the PELARS project system and introduced to their first task. Task 1 required students to design and prototype an interactive toy. Task 2 required students to design and prototype a colour sorting machine, and Task 3 required students to design and prototype an autonomous automobile. No specific instructions about the timing of these phases were given to students and sessions lasted between 33 and 75 minutes (with the median of 63 minutes).

\subsection{Data Collection and Analysis}

All sessions were video recorded through the PELARS learning analytics system. This video data was analysed by two researchers using a very simple coding scheme, which was designed to inform future automatic video analysis.

The coding scheme makes use of three digits, 0,1 and 2 to represent passive, semiactive and active student physical states, respectively. The active code (2) was used whenever a student's hand was active with an object; the semi-active code (1) was used when a student was not physically active but their head was directed towards a peer who was active, or to the object he/she was manipulating, or to the screen; and the passive code (0) was used if a students hands were not physically active with any object and their head was directed towards a different position than any of their peers who were active. Students behaviours were coded using thirty seconds windows. Therefore the variable used for our research questions is the activity index $A I$, which takes values $0,1,2$ and is defined in equation 1 :

AI $(S, G, t):=$ Activity code of student $S$ of group $G$ at time $t$

where $S=1,2,3 ; G=A, \ldots, L$ and $t=30,60,90 \ldots$

For example, in the situation represented by the photo shown in Figure 1, the student to the left is coded as 2 , while the other two students are coded as 1 . To validate the coding, two coders applied this coding scheme to all groups video data using 30-second windows. Where there was disagreement, the researchers discussed the data and revised their coding accordingly. Thanks to the simplicity of the coding scheme, there were only a few differently coded 30s windows, and these were resolved easily through discussion. This discussion involved playing the window again together to decide on the final code.

\subsection{Observer Analysis of Collaborative Problem Solving Competencies}

In addition to the data capture facilitated by the project system, human observers analysed student interactions as they happened using an analysis framework [27], based on OECDs work on CPS [28]. The analysis framework has 


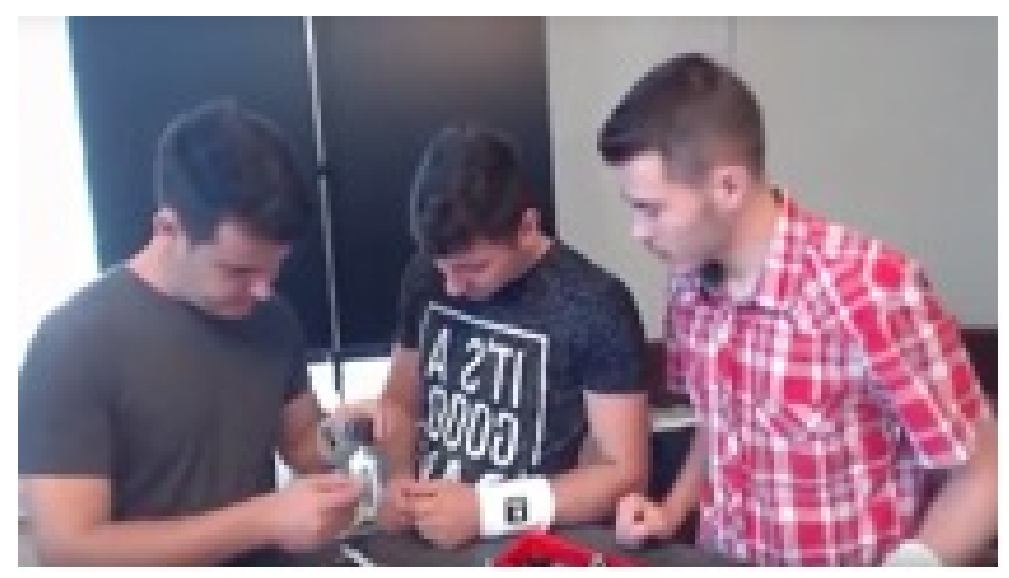

Fig. 1. A moment during students' work to exemplify the coding scheme

Table 1. Classification of students' groups according to their level of CPS

\begin{tabular}{cr}
\hline Group Code & Level of CPS Competency \\
\hline A & Medium \\
B & Low \\
C & Medium \\
D & High \\
E & Medium \\
F & High \\
G & Medium \\
H & Medium \\
I & Medium \\
J & High \\
K & Low \\
L & Medium \\
\hline
\end{tabular}


three key dimensions of collaboration (Establishing and maintaining shared understanding, Taking appropriate actions to solve the problem, Establishing and maintaining team organisation), and six key dimensions of problem-solving (Identifying facts, Representing and formulating knowledge, Generating hypotheses, Planning and executing, Identifying knowledge and skill deficiencies, Monitoring, reflecting and applying). The human observer watches student activity and uses a mobile tool to mark the critical incidents that relate to the key dimensions of collaboration and problem solving as they occur [29]. The tool also records the exact date and time each dimension was marked by the human observer. Using this framework student groups were ranked as high, medium and low-level collaboration groups. The ranking was done based on threshold values of the frequency of critical incidents, and this ranking was used as an independent variable for the analysis presented in this paper.

\section{Results}

Table 1 shows the classification of the different groups using the observer coding. Groups D, F and J were coded as the highest CPS groups, whereas B and K were coded as the lowest CPS groups.

\subsection{Equality of Students Physical Interactivity}

In order to answer our first research question, we first investigated the extent to which the degree of equality observable in the students physical interactivity can be used as a non-verbal index to interpret collaboration in practice-based learning activities. To this end, we defined new research variables (equation 2):

$$
N j(G, i):=\text { Percentage of } i \text { states for student } j \text { in group } G
$$

where $i, j=1,2,3$ and $G=A, \ldots, L$

Figure 2 presents the coding of each students physical interactivity and illustrates that some groups showed more equality (e.g. groups I, J, D) than others. The distribution is irregular (e.g. groups B, E) and identifies the students who were more engaged (e.g, student S1 in group F) and students who were less engaged (e.g student 1 in the A group). In order to have a better idea about the equality of students physical interactivity, we looked at the mean scores of their codes. Table 2 presents these results and indicates in dark grey the groups which were identified by the observer as high CPS groups. The groups identified as lower CPS are indicated by a lighter shade of grey.

As the results above show, those groups coded as high collaboration groups by human observers had higher mean scores for physical interactivity than those coded as low collaboration groups. Considering the practice-based structure of the learning activity these results are not surprising. However, a finding that becomes clear from table 2 is that the groups rated as high collaboration groups have member students whose physical interactivity mean scores are similar. By 

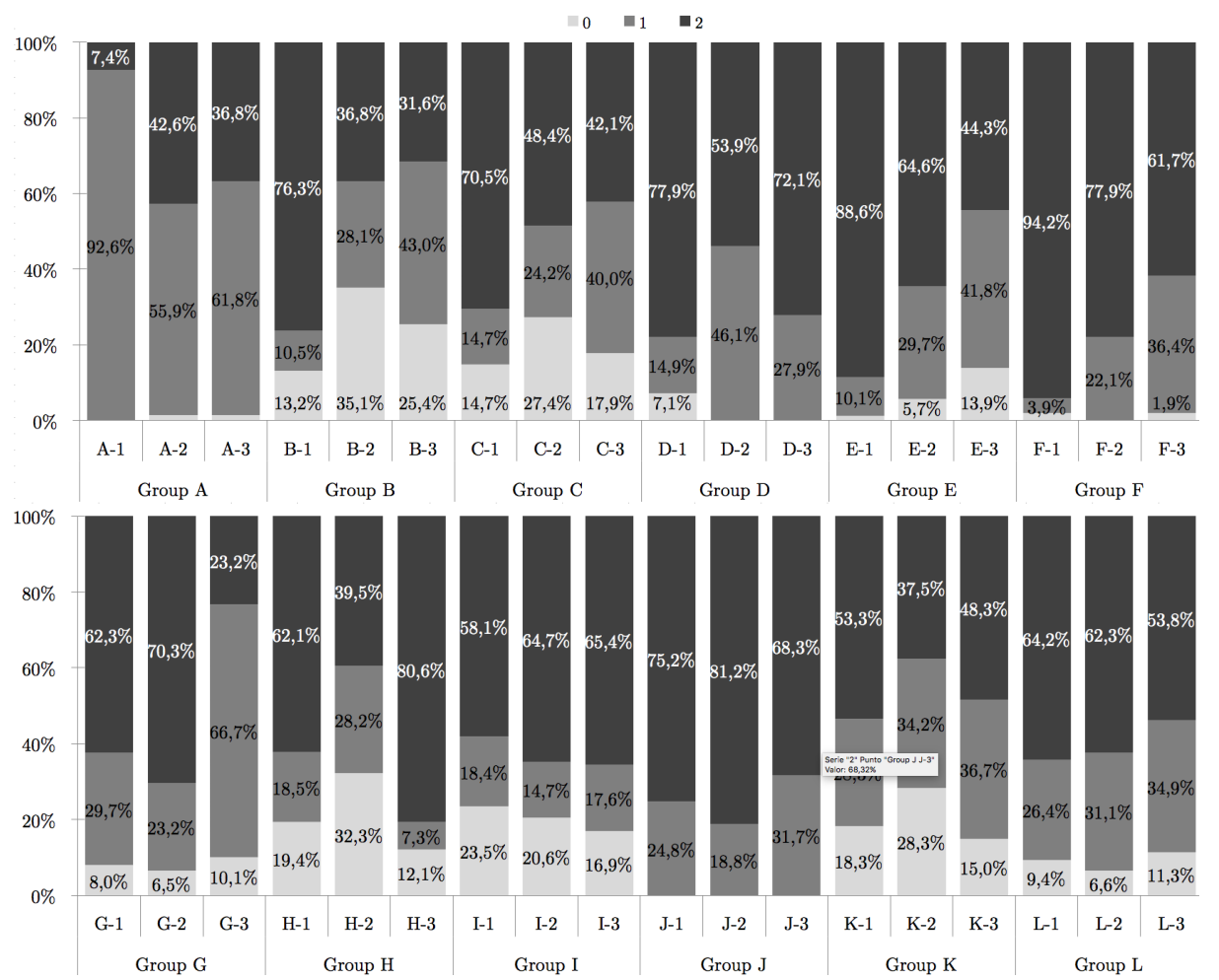

Fig. 2. Percentages of individual student's number of passive 0 , semi-active 1, and active codes 2 


\begin{tabular}{|c|c|c|c|c|c|c|c|c|c|c|c|c|c|c|c|c|c|c|}
\hline & \multicolumn{3}{|c|}{ Group A } & \multicolumn{3}{|c|}{ Group B } & \multicolumn{3}{|c|}{ Group C } & \multicolumn{3}{|c|}{ Group D } & \multicolumn{3}{|c|}{ Group E } & \multicolumn{3}{|c|}{ Group F } \\
\hline & A.s1 & A.s2 & A.s3 & B.s1 & B.s2 & B.s3 & C.s1 & C.s2 & C.s3 & D.s1 & D.s2 & D.s3 & E.s1 & E.s2 & E.s3 & F.s1 & F.s2 & F.s3 \\
\hline Mean AI & 1,07 & 1,41 & 1,35 & 1,71 & 1,07 & 1,12 & 1,63 & 1,29 & 1,33 & 1,70 & 1,60 & 1,75 & 1,87 & 1,60 & 1,31 & 1,92 & 1,78 & 1,60 \\
\hline Sd AI & 0,26 & 0,53 & 0,51 & 1,71 & 1,07 & 1,12 & 0,64 & 0,78 & 0,65 & 0,61 & 0,49 & 0,43 & 0,37 & 0,59 & 0,70 & 0,33 & 0,42 & 0,53 \\
\hline Av. Mean & \multicolumn{3}{|c|}{1,28} & \multicolumn{3}{|c|}{1,30} & \multicolumn{3}{|c|}{1,41} & \multicolumn{3}{|c|}{1,68} & \multicolumn{3}{|c|}{1,59} & \multicolumn{3}{|c|}{1,77} \\
\hline Max. diff. & \multicolumn{3}{|c|}{0,68} & \multicolumn{3}{|c|}{1,27} & \multicolumn{3}{|c|}{0,68} & \multicolumn{3}{|c|}{0,31} & \multicolumn{3}{|c|}{1,12} & \multicolumn{3}{|c|}{0,65} \\
\hline & \multicolumn{3}{|c|}{ Group G } & \multicolumn{3}{|c|}{ Group H } & \multicolumn{3}{|c|}{ Group I } & \multicolumn{3}{|c|}{ Group J } & \multicolumn{3}{|c|}{ Group K } & \multicolumn{3}{|c|}{ Group L } \\
\hline Mean AI & G.s1 & G.s2 & G.s3 & H.s1 & H.s2 & H.s3 & I.s1 & I.s2 & I.s3 & J.s1 & J.s2 & J.s3 & K.s1 & K.s2 & K.s3 & L.s1 & L.s2 & L.s3 \\
\hline Sd AI & 1,54 & 1,64 & 1,13 & 1,45 & 1,10 & 1,71 & 1,43 & 1,55 & 1,58 & 1,75 & 1,81 & 1,68 & 1,35 & 1,09 & 1,33 & 1,55 & 1,56 & 1,42 \\
\hline Av. Mean & 0,64 & 0,60 & 0,56 & 0,77 & 0,83 & 0,63 & 0,77 & 0,72 & 0,67 & 0,43 & 0,39 & 0,47 & 0,77 & 0,81 & 0,73 & 0,66 & 0,62 & 0,69 \\
\hline Max. diff. & \multicolumn{3}{|c|}{1,44} & \multicolumn{3}{|c|}{1,42} & \multicolumn{3}{|c|}{1,52} & \multicolumn{3}{|c|}{1,75} & \multicolumn{3}{|c|}{1,26} & \multicolumn{3}{|c|}{1,51} \\
\hline & \multicolumn{3}{|c|}{1,01} & \multicolumn{3}{|c|}{1,23} & \multicolumn{3}{|c|}{0,29} & \multicolumn{3}{|c|}{0,26} & & 0,52 & & & 0,26 & \\
\hline
\end{tabular}

Table 2. Mean activity index per student, standard deviations, average mean and total mean differences

contrast, the groups rated as low collaboration groups have member students whose mean scores for the physical interactivity of each student are more varied. For instance, in group D, which was coded as a high collaboration group, the mean scores for the member students physical interactivity were $s 1=1.60$, $\mathrm{s} 2=1.70$, and $\mathrm{s} 3=1.75$; and the average of differences between the three students physical interactivity scores was 0.31 . On the other hand, the mean physical interactivity scores for member students of group B, which was coded as one of the low collaboration groups, were $\mathrm{s} 1=1.07, \mathrm{~s} 2=1.12$, and $\mathrm{s} 3=1.71$ and the average of the differences between the three students physical interactivity was 1.27. The difference in physical interactivity scores for group B is approximately four times bigger than the average differences in the high collaboration group D. These results suggest that equality of students physical interactivity is a potential indicator of collaboration in practice-based learning activities. It is important to note that when the students physical interactivity is low, for instance, as in the case of the group K, which had the lowest average mean score for physical interactivity among all groups, then the ratio of the differences in scores by member students between low collaboration and high collaboration groups does not hold. The level of activity is too low.

\subsection{Intra-individual variability of students physical interactivity}

The second potential non-verbal indicator of collaboration we investigated is the intra-individual variability of students physical interactivity. Intra-individual variability refers to the amount of change in every single students behaviour between two sequential time windows. The cause of these changes were not taken into account and we used a simple statistical formula to calculate it as the mean sequential squared difference $M$. This formula is presented in equation 3 :

$$
M:=\frac{1}{N-1} \sum_{k=1}^{N-1}\left(x_{k-1}-x_{k}\right)^{2}
$$


We consider $M$ as a good method to calculate students intra-individual variability, as it represents the mean value of the total amount of changes in students physical interactivity. Table 3 shows the computed $M$ for each student, together with the total group differences. Then the total differences values $T$ are calculated by summing the differences of three students $M$ values using the formula defined in equation 4 .

$$
T:=\left(M_{\max }-M_{m i d}\right)+\left(M_{\max }-M_{\min }\right)+\left(M_{m i d}-M_{\min }\right)
$$

\begin{tabular}{|c|c|c|c|c|c|c|c|c|c|c|c|c|c|c|c|c|c|c|}
\hline & \multicolumn{3}{|c|}{ Group A } & \multicolumn{3}{|c|}{ Group B } & \multicolumn{3}{|c|}{ Group C } & \multicolumn{3}{|c|}{ Group D } & \multicolumn{3}{|c|}{ Group E } & \multicolumn{3}{|c|}{ Group F } \\
\hline & A.s1 & A.s2 & A.s3 & B.s1 & B.s2 & B.s3 & C.s1 & C.s2 & C.s3 & D.s1 & D.s2 & D.s3 & E.s1 & E.s2 & E.s3 & F.s1 & F.s2 & F.s3 \\
\hline 1010 & & 0.61 & 0.83 & 52 & 0.61 & 0.84 & 0.68 & 0.89 & 0.51 & 0.29 & 0.27 & 0.24 & 0.27 & 0.53 & 0.42 & 0.20 & .25 & 0.38 \\
\hline \multirow[t]{3}{*}{ Total diff. } & \multicolumn{3}{|c|}{0.57} & \multicolumn{3}{|c|}{0.64} & \multicolumn{3}{|c|}{0.58} & \multicolumn{3}{|c|}{0.09} & \multicolumn{3}{|c|}{0.51} & \multicolumn{3}{|c|}{0.33} \\
\hline & \multicolumn{3}{|c|}{ Group G } & \multicolumn{3}{|c|}{ Group H } & \multicolumn{3}{|c|}{ Group I } & \multicolumn{3}{|c|}{ Group J } & \multicolumn{3}{|c|}{ Group K } & \multicolumn{3}{|c|}{ Group L } \\
\hline & G.s1 & G.s2 & G.s3 & H.s1 & H.s2 & H.s3 & I.s1 & I.s2 & I.s3 & \begin{tabular}{|l|} 
J.s1 \\
\end{tabular} & J.s2 & J.s3 & K.s1 & K.s2 & K.s3 & L.s1 & L.s2 & L.s3 \\
\hline 1010 & 0.60 & 0.41 & 0.63 & 0.73 & 0.76 & 0.45 & 0.77 & 0.58 & 0.72 & 0.22 & 0.22 & 0.27 & 0.98 & 0.92 & 0.43 & 0.82 & 0.60 & 0.54 \\
\hline Total diff. & \multicolumn{3}{|c|}{0.44} & \multicolumn{3}{|c|}{0.63} & \multicolumn{3}{|c|}{0.38} & \multicolumn{3}{|c|}{0.10} & \multicolumn{3}{|c|}{1.11} & \multicolumn{3}{|c|}{0.55} \\
\hline
\end{tabular}

Table 3. $M$ values per group and student and group $M$ differences

Results show that high collaboration groups show lower $M$ values, whereas low collaboration groups show higher $M$ values. If we look at the average differences of individual students $M$ scores, high collaboration groups appear to have the smallest three figures (Group $\mathrm{D}=0.09$, Group $\mathrm{F}=0.33$, Group $\mathrm{J}=$ 0.10 ), whereas low collaboration groups have the highest two figures (Group $\mathrm{B}=$ 0.64, Group $\mathrm{K}=1.11$ ). The low $M$ values can be achieved if students continue their level of physical interactivity for longer periods of times, rather than having frequent changes in their interactivity. Figure 3 illustrates the chronological changes in $M$ value for Group F, assessed as being a high CPS group and Group $\mathrm{K}$, assessed as a low CPS group.

One potential explanation for continuing on the same action is that, students have a sense of mutual understanding of the task/problem they are working on. When such mutual understanding does not occur among group members, their actions may appear to vary more often as they stop and start their physical activities more frequently. The importance of mutual understanding as a dimension of collaboration has been recognized by other researchers (e.g. [30], [31], [32]). And the magnitude of change in physical interactivity measurement may be one option to interpret this mutual understanding. Our results suggest that the intra-individual variability of students physical interactivity is another potential indicator of collaborative problem solving in practice-based learning activities.

\section{Discussion}

Students knowledge acquisition is important. However, students must also be able to apply this knowledge, to explain it clearly to others, to synthesize it with 


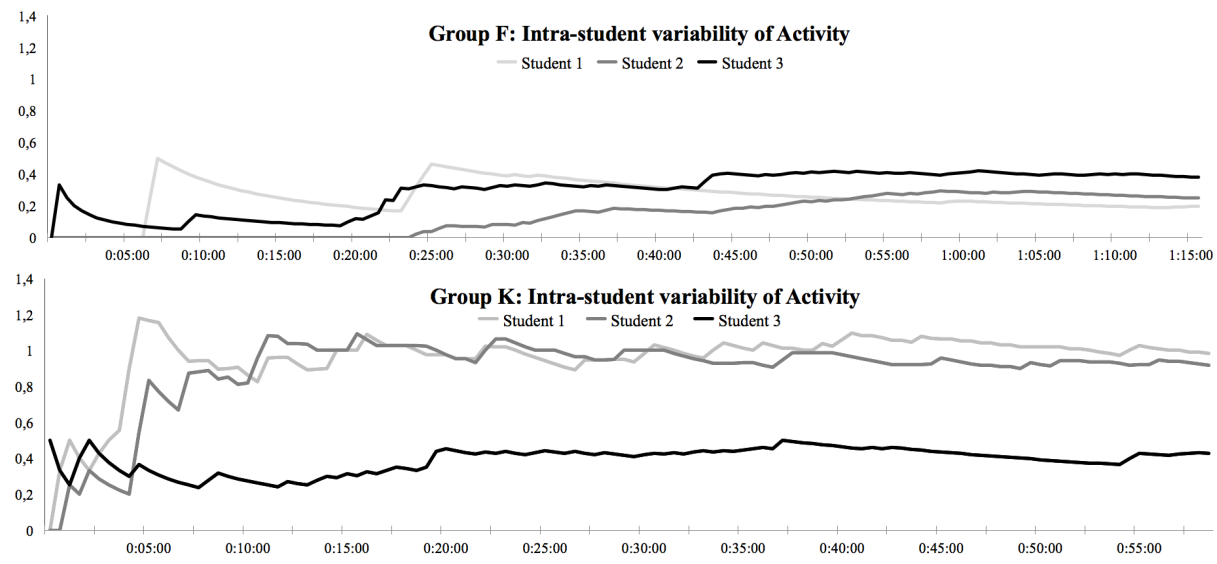

Fig. 3. Chronological changes of $M$ in high and low CPS groups

newly acquired knowledge from the same or other subject areas. They must also be able to use their knowledge to solve problems collaboratively. Subject specific knowledge and routine cognitive skills are the easiest to be automated with technology and these alone are no longer enough in the modern workplace. As science and technology continue to progress the notion of a body of knowledge will increasingly be something that will be distributed amongst multiple intelligences, both human and machine. It is therefore of great importance that young people should acquire appropriate collaboration skills to be able to solve problems and tasks that neither of the multiple agencies (including human and machine) of the future would be able to solve on their own.

However, acquisition of CPS skills requires purposeful practice of collaboration in settings that differ from uncollaborative group work or other peer learning settings including cooperation and peer tutoring approaches. Hence, the identification of indicators of collaborative problem-solving and their support have great importance to the researchers and practitioners of the research community. In this paper, we investigate the potential of two non-verbal indexes of students physical interactivity, to identify the level of collaboration in groups of students. Our first research question was: Can equality of physical interactivity and intra-individual variability be used as non-verbal indexes of collaboration in practice-based learning? Our results show that both the measures of students equality of physical interactivity and measures of intra-individual variability are useful indexes of students physical interactivity that can be used to evaluate the level of collaboration in a group. Related to this question our second research question was: What amounts of physical interactivity and intra-individual variability represent collaboration in practice-based learning? Analysing the data from twelve groups of Engineering students working in groups of three in openended practice-based activities, we found that students in groups that had been evaluated by a human observer as being high collaboration groups have member 
students who have high and equal scores for physical interactivity and low and equal scores for intra-individual variability.

These results are aligned with the existing research findings in the field. For instance, earlier research on peer learning shows that collaborative groups are high in equality and mutuality [7], they move in unison [33], [34], they present symmetry in terms of their status and contributions [35], and they are synchronised in their gaze [36]. In addition to these concepts, we argue that, students intra-individual variability in their physical interactivity is an important indicator of collaboration in practice-based learning and it may reflect the mutual understanding between students in a group.

We must point out the limitations of this work as well as its potential benefits. The evaluation of student performance through concepts such as, equality and intra-individual variability is only one small part of understanding how good a student is at CPS. The CPS process is much more complicated than any of the existing statistical measures of performance, particularly when it comes to complex learning environments of practice-based learning. However, these statistical measurements can act as useful indicators of potential success or failure of collaboration. Although our results are derived from twelve groups of three students, which can be considered as a small sample size, they are promising and we aim to investigate them further with larger sample sizes.

\section{Conclusions}

In this research paper, we present two non-verbal indexes of students physical interactivity that can be used to interpret the collaborative nature of their practice-based activities. Students in collaborative problem-solving groups show high levels of physical interactivity and low levels of intra-individual variability. Both of these indexes present smaller ranges in high collaboration groups when compared with low collaboration groups. Our simple coding scheme of students active, semi-active and passive positions is a practical and valuable approach that can inform the design of automated analysis systems. Hence, future research could involve attempts to automate this process of coding and provide real-time feedback to students and teachers about the collaborative or non-collaborative patterns of their physical interactivity during their learning activities. These results would have multiple implications both for the design and implementation of peer learning activities in classrooms and they would increase the accuracy and timeliness of teacher interventions. We argue that the most effective and efficient education can be provided through combining the statistical analyses of student performances with teachers expert instinctive judgment of the learning situations. Nevertheless, it would be wrong to rely only on such instinctive judgment, in the same way that it would be wrong to rely only on similar statistical calculations to these presented in this paper. 


\section{Acknowledgements}

This work is co-funded by the European Union under the PELARS project. Third author was partially supported by Agencia Estatal de Investigación (AEI) y el Fondo Europeo de Desarrollo Regional (FEDER), TIN2016-80774-R.

\section{References}

1. Luckin, R., Baines, E., Cukurova, M., Holmes, W.: Solved! Making the case for collaborative problem-solving. A report for Nesta. Nesta, London, UK. (2017)

2. Johnson, D.W., Johnson, R.T.: Learning together and alone: Overview and metaanalysis. Asia Pacific Journal of Education 22(95-105) (2002)

3. Kyndt, E., Raes, E., Lismont, B., Timmers, F., Cascallar, E., Dochy, F.: A metaanalysis of the effects of face-to-face cooperative learning: Do recent students verify or falsify earlier findings? Educational Research Review 10 (2013) 133-149

4. Nunnery, J.A., Chappel, S., Arnold, P.: A meta-analysis of a cooperative learning model: Effects on student achievement in mathematics. Cypriot Journal of Educational Sciences 8(1) (2013) 34-48

5. Puzio, K., Collby, G.T.: Cooperative learning and literacy: A meta-analytic review. Journal of Research on Educational Effectiveness 6(4) (2013) 339-360

6. Roseth, C.J., Johnson, D.W., Johnson, R.T.: Promoting early adolescents' achievement and peer relationships: the effects of cooperative, competitive, and individualistic goal structures. Psychological Bulletin 134(2) (2008) 223

7. Damon, W., Phelps, E.: Critical distinctions among three approaches to peer education. International Journal of Educational Research 13(1) (1989) 9-19

8. Dillenbourg, P.: What do you mean by 'collaborative learning'? Cognitive and Computational Approaches (1999) 1-19

9. Sharan, S.: Cooperative learning in small groups: Recent methods and effects on achievement, attitudes, and ethnic relations. Review of educational research $\mathbf{5 0}(2)$ (1980) 241-271

10. Clarke, J.: Pieces of the puzzle: The jigsaw method. Handbook of cooperative learning methods. Pearson, London (1994)

11. Sharan, S.: Cooperative learning: Theory and research. Praeger Publishers, USA (1990)

12. Kirschner, F., Paas, F., Kirschner, P.A., Janssen, J.: Differential effects of problemsolving demands on individual and collaborative learning outcomes. Learning and Instruction 21(4) (2011) 587-599

13. Kirschner, F., Paas, F., Kirschner, P.A.: Task complexity as a driver for collaborative learning efficiency: The collective working-memory effect. Applied Cognitive Psychology 25(4) (2011) 615-624

14. Metcalf, S., Kamarainen, A., Tutwiler, M.S., Grotzer, T., Dede, C.: Ecosystem science learning via multi-user virtual environments. International Journal of Gaming and Computer-Mediated Simulations (IJGCMS) 3(1) (2011) 86-90

15. Rouet, J.F.: The skills of document use. Erlbaum, Mahwah, NJ (2006)

16. Spikol, D., Avramides, K., Cukurova, M., Vogel, B., Luckin, R., Mavrikis, M., Ruffaldi, E.: Exploring the interplay between human and machine annotated multimodal learning analytics in hands-on stem activities. Proceedings of the 6th International Learning Analytics \& Knowledge Conference (2016) 
17. Trilling, B., Fadel, C.: 21st century skills: Learning for life in our times. John Wiley and Sons, USA (2009)

18. WEF: New vision for education: Fostering social and emotional learning through technology. World Economic Forum: Industrial Agenda Report (2016)

19. Nevin, S.: Lifelong learning: Reforming education for an age of technological and demographic change. Institute of Directors Policy Report March 2016

20. Dillenbourg, P., Lemaignan, S., Sangin, M., Nova, N., Molinari, G.: The symmetry of partner modelling. International Journal of Computer-Supported Collaborative Learning 11(2) (2016) 227-253

21. Blech, C., Funke, J.: Dynamis review: An overview about applications of the dynamis approach in cognitive psychology. Bonn: German Institute for Adult Education (DIE) (2005)

22. Wirth, J., Klieme, E.: Computer-based assessment of problem solving competence. Assessment in Education: Principles, Policy, and Practice 10(3) (2004) 329-345

23. Clark, R.E.: Using language. Cambridge University Press, Cambridge (1996)

24. Marlowe, H.A.: Social intelligence: Evidence for multidimensionality and construct independence. Journal of educational psychology 78(1) (1986) 52-58

25. Argyle, M. In: New developments in the analysis of social skills. Academic Press, New York (1979) 139-158

26. Vallacher, R.R., Read, S.J., Nowak, A.: The dynamical perspective in personality and social psychology. Personality and Social Psychology Review 6(4) (2002) 264273

27. Cukurova, M., Avramides, K., Spikol, D., Luckin, R., Mavrikis, M.: An analysis framework for collaborative problem solving in practice-based learning activities: a mixed-method approach. In: Proceedings of the International Conference on Learning Analytics and Knowledge, Edinburgh, United Kingdom, ACM (2016) $84-88$

28. OECD: Draft collaborative problem solving framework. Report (2015)

29. Cukurova, M., Avramides, K., Luckin, R., Mavrikis, M.: Revealing behaviour pattern differences in collaborative problem solving. In: Adaptive and Adaptable Learning. 11th European Conference on Technology Enhanced Learning (EC-TEL 2016), Switzerland, Springer (2016) 563-569

30. Andriessen, J., Baker, M., Suthers, D.: Arguing to learn: Confronting cognitions in computer-supported collaborative learning environments. Volume 1. Springer Science \& Business Media (2013)

31. Barkley, E.F., Cross, K.P., Major, C.H.: Collaborative learning techniques: A handbook for college faculty. John Wiley \& Sons (2014)

32. Engeström, Y.: Learning by expanding. Cambridge University Press (2014)

33. Lakens, D.: Movement synchrony and perceived entitativity. Journal of Experimental Social Psychology 46(5) (2010) 701-708

34. Lakens, D., Stel, M.: If they move in sync, they must feel in sync: Movement synchrony leads to attributions of rapport and entitativity. Social Cognition 29(1) (2011) 1-14

35. Dillenbourg, P., Zufferey, G., Alavi, H., Jermann, P., Do-Lenh, S., Bonnard, Q.: Classroom orchestration: The third circle of usability. In: International Conference on Computer Supported Collaborative Learning 2011 (CSCL 2011), ISLS 510-517

36. Schneider, B., Pea, R.: Real-time mutual gaze perception enhances collaborative learning and collaboration quality. International Journal of Computer-Supported Collaborative Learning 8(4) (2013) 375-397 